\title{
Evaluation between methods for the color measurement in holograms by using a CMOS-RGB camera and a spectrometer
}

\author{
Natalith Palacios-Ortega ${ }^{*^{a}}$, Daniel Velásquez Prieto ${ }^{a}$ \\ ${ }^{a}$ Grupo de Óptica Aplicada, Departamento de Ciencias Básicas, Universidad EAFIT, Medellín \\ (7023), Colombia
}

\begin{abstract}
Many models and methods commonly used in colorimetry have been incorporated to the study and knowledge of the colorimetric properties in the reflection color holograms; these methods have reported the possibilities of color reproduction in holograms. One method is based in calculating the color differences between the CIE-L*a*b* coordinates of the original object compared to the same values obtained for the reconstructed hologram; these values are calculated through the measurement of the spectral composition of the light in the reproduced hologram which are made with spectrometers. Other methods are based in the use of cameras for the color measurement, although, they are not commonly used for that ending in holography. This work presents the results of a comparative study between the use of spectrometers and RGB digital cameras for the color measurement in holograms. The diffraction efficiency of the holograms for a GretagMacbeth Colorchecker samples is measured through a spectrometer and their CIE-L*a*b* coordinates are calculated; the color differences are also calculated by taking as theoretical values the coordinates of the original object. A similar procedure is made by capturing the reconstructed images of the hologram through a CMOSRGB camera, which requires a linearizing and characterizing procedure. The RGB coordinates of the original object are compared with the RGB coordinates of the reproduced hologram too.
\end{abstract}

Keywords: CIE-XYZ, CIE-L*a*b*, color reflection hologram, spectrometer, CMOS-RGB camera, diffraction efficiency.

\section{INTRODUCTION}

The advancing of the methods for measuring the color reproducibility in holography have gained importance with the developing of new techniques, materials and equipment for the color holograms recordings, examples of those are the manufacturing of lasers which have available three primary wavelengths that are necessary for obtaining color (blue, green and red), panchromatic register materials and more recently because of the developing of newer color holographic displays, which is the case of the holographic stereoscopy.

In color holography, the images are registered through three lasers with different wavelengths (RGB) through an interference process and on its reproduction the image is obtained as the result of a diffraction process of discretized light sources, like lasers, RGB LEDs, or white light sources. By means of this procedure the color not only depends of the spectral composition of the light source but also of the characteristics of the diffraction grids which conform the hologram, while in the visualization and capturing of $2 \mathrm{D}$ images, the color is obtained and measured through the absorption and reflection phenomena.

Many authors have proposed diverse methods which are mainly based in models that classically have been used in colorimetry and employ instruments like spectrometers, colorimeters and spectroradiometers ${ }^{1-3}$. By now, digital RGB cameras have been used like colorimeters ${ }^{4}$; however, its use like a color measurement instrument has been not quite studied. Anyhow, the case of interest is calculating the ability to reproduce the color or light source.

In this work, a comparative study is made between the use of spectrometers and RGB digital cameras for the color measurement in holograms by calculating their chromatic reproduction index and color difference; the first one is determinated by the distance between the chromaticity coordinates $\left(a^{*}\right.$ and $\left.b^{*}\right)$ of the original object and the same coordinates for the reproduced image, this information is used to draw some vectors in the plane $\mathrm{a}^{*}, \mathrm{~b}^{*}$; the second one is calculated not only with the chromaticity coordinates but also with the luminance coordinate.

*npalaci4@eafit.edu.co; phone 5742619500 extension 9644; fax 5742664284

8th Iberoamerican Optics Meeting and 11th Latin American Meeting on Optics, Lasers, and Applications,

edited by Manuel Filipe P. C. Martins Costa, Proc. of SPIE Vol. 8785,878568

(C) 2013 SPIE $\cdot$ CCC code: 0277-786X/13/\$18 · doi: 10.1117/12.2027569 


\section{COLOR MEASUREMENT IN HOLOGRAMS}

The advancing in the methods for measuring the color reproducibility in holography have gained importance with the developing of new techniques, materials and equipment for the color holograms recordings, examples of those are the manufacturing of lasers which have available three primary wavelengths that are necessary for obtaining color (blue, green and red), panchromatic register materials and more recently because of the developing of newer color holographic displays, which is the case of the holographic stereoscopy.

\subsection{Color measurement in holograms with spectrometer}

A common method used for color measurement is based in calculating the CIE-L*a*b* color coordinates which can be obtained by transforming the XYZ values, they represent lineal combinations between the reflectance of the sample $P(\lambda)$, the spectral distribution of the employed illuminant $E(\lambda)$ and the color matching values $x(\lambda), y(\lambda), z(\lambda)$; so, XYZ values and CIE-L*a*b* values can be given $b y^{5}$ :

$$
X=k \sum_{400 \mathrm{~nm}}^{700 \mathrm{~nm}} E(\lambda) P(\lambda) x(\lambda) \quad Y=k \sum_{400 \mathrm{~nm}}^{700 \mathrm{~nm}} E(\lambda) P(\lambda) y(\lambda) \quad Z=k \sum_{400 \mathrm{~nm}}^{700 \mathrm{~nm}} E(\lambda) P(\lambda) z(\lambda)
$$

In this equation, $P(\lambda), E(\lambda)$ are measured with a spectrometer, $x(\lambda), y(\lambda), z(\lambda)$ are pre-established values for color measurement. The transformation between XYZ and CIE-L*a*b* is given by:

$$
\begin{aligned}
& L^{*}=116 f\left(Y / Y_{n}\right)-16 \\
& a^{*}=500\left[f\left(X / X_{n}\right)-f\left(Y / Y_{n}\right)\right] \quad b^{*}=200\left[f\left(Y / Y_{n}\right)-f\left(Z / Z_{n}\right)\right] \\
& \text { If } \omega>0.008856 \quad f(\omega)=\omega^{1 / 3} \quad \text { If } \omega<0.008856 f(\omega)=7.787 \omega+(16 / 116)
\end{aligned}
$$

Where $\mathrm{k}$ is a normalization constant and $\mathrm{Xn}, \mathrm{Yn}, \mathrm{Zn}$ are the $\mathrm{XYZ}$ values of the illuminant.

The color difference $\Delta \mathrm{E}$ is a comparison made between the experimental CIE-L*a*b* values of the sample and its theoretical values, it means that if the sample is acquired through a camera, scanner or illuminated under determinate light source, its color coordinates would change; that is why these coordinates have to be compared with its original or theoretical coordinates. The color difference can be represented in a diagram by drawing its experimental $\mathrm{a}^{*}$ and $\mathrm{b}^{*}$ coordinates as a point on the plane and taking them to another point $a^{*}$ and $b^{*}$ which are the theoretical values; it will form a vector, if its magnitude increase, it will represent a high color difference which means a poor reproducibility, otherwise, if the magnitude is small, the instrument (RGB camera, scanner, or any kind of light source) has a high reproducibility. The color difference is given by:

$$
\Delta E=\sqrt{\Delta L^{2}+\Delta a^{2}+\Delta b^{2}}
$$

The color measurement methods based in spectrometers were the first in being used for color measurement in holograms ${ }^{3}$; this was necessary because of the advances presented in color holography, which required a method to verify quantitatively the reproducibility of the reconstructed hologram. The employed methods are based in spectrometers, colorimeters and in models that search for calculating the color coordinates and the color differences $\Delta \mathrm{E}$. Basically, the usual configuration uses a sensor which measures the reflectance of the sample, in this case, is the diffraction efficiency of the hologram, a source light that represents a standard illuminant or a source that reproduces the hologram and an algorithm that calculate the values of (1), (2) and (3). Many authors have used this method ${ }^{1-3}$, some of them in order to know the adequate wavelengths for the recording of color holograms, and others to know the reproducibility of the recording method $^{6-8}$.

\subsection{Color measurement in holograms with RGB camera}

RGB cameras can be used as colorimeters too, because of the three values that they give as an answer of the three filters that conform the color in this type of instruments; however, the RGB values that a camera gives are not the same XYZ values that a colorimeter would usually present; also, the answer of a RGB camera is not lineal because of nonlinearities that the manufacturer introduces in the instrument looking for to improve the signal to noise ratio; that is why a linearization process is needed in the camera, after that, a characterization or transformation is made to convert the RGB values to $\mathrm{XYZ}^{9}$. 
A linearization procedure of a RGB camera consists in generating a linear answer of the RGB channels of the camera according to the light that arrives to the sensor ${ }^{10}$, so, by measuring the reflectance $\mathrm{P}(\lambda)$ of determined neutral samples (gray samples), the answer of the sensor without linearization would be represented by:

$$
R^{\prime}=P^{\gamma} \quad G^{\prime}=P^{\gamma} \quad B^{\prime}=P^{\gamma}
$$

Here, $\gamma$ is the non-linearity exponent of each one of the channels and that is the value to find out in order to perform the correction of the non-linearity; these exponents are found through the minimum square method.

Once the camera is linearized, a characterization is required, which means to find a lineal or polynomial transformation that converts the RGB values of the camera to XYZ for color measurement, this transformation is given by a matrix which is found by means of a mapping of known XYZ values and their corresponding RGB values given by a camera. That is:

$$
A=D^{+} T
$$

Here, $\mathrm{D}^{+}$is the pseudoinverse matrix of the RGB values which are given by the camera and $\mathrm{T}$ is the $\mathrm{XYZ}$ matrix which values are known by means of a spectrometer. The $\mathrm{D}$ matrix could be linear or polynomial relationships of the way ${ }^{9-10}$ :

$$
\begin{gathered}
R \quad G \quad B \\
R \quad G \quad B \quad R G \quad R B \quad G B 1 \\
R \quad G \quad B \quad R G \quad R B \quad G B R^{2} G^{2} B^{2} 1
\end{gathered}
$$

\section{EXPERIMENTAL ARRANGEMENT}

\subsection{Holograms Register}

An experimental arrangement was implemented for recording reflection holograms of single beam, a superior view of the configuration is shown in Figure (1) a. This arrangement uses a coherent RGB illumination system compound by three lasers: R (He-Ne), G (DPSS) and B (DPSS), which correspond to the wavelengths of $632,8 \mathrm{~nm}, 532 \mathrm{~nm}$ and $473 \mathrm{~nm}$ respectively. The control of the transmitted intensity is made individually for each wavelength by means of variable neutral density filters (NDF) which are located at the exit of each one of the lasers. The mixing of the three wavelengths is made using the M1 mirror and two dichroic filters (DF1 and DF2); this produces at the exit a coherent beam of "white" light of RGB components. With the mirrors M3a and M3b the beam is guided to obtain an illumination on the plate and above the object, forming an angle $\theta$ as seen in Figure (1) b. The recording was made in emulsions of ultrafine grain Slavich PFG-03C and was revealed with $\mathrm{CW}-\mathrm{C} 2^{11}$.

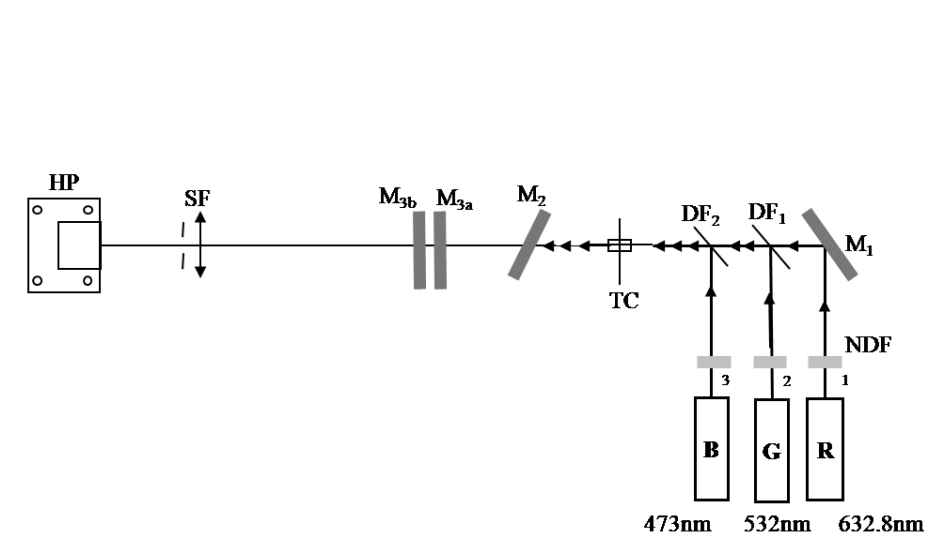

(a)

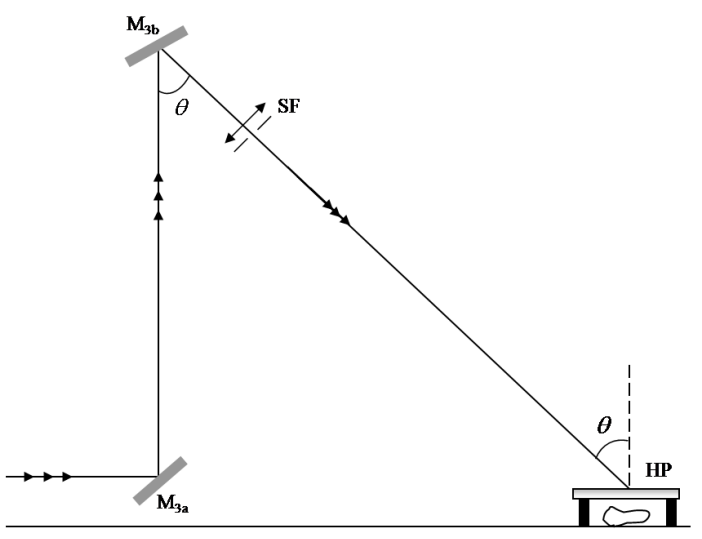

(b)

Figure 1. Scheme for recording reflection holograms of single beam ${ }^{11}$ (a) Upper View (b) Side View 


\subsection{Color measurement arrangement with spectrometer}

The arrangement for measuring color with spectrometer consisted in a light source which had three RGB lasers, the hologram was the sample to be measured; a Newport IS Series Minispectrometer was used as the sensor and a fiber optic arrangement coupled to it helped to capture the diffracted light of the sample. To make the measurements, the hologram was illuminated to a $30^{\circ}$ angle, just the same angle used for the recording; this arrangement is detailed in Figure (2). The registered and analyzed object was the Macbeth Colorchecker Mini ${ }^{\circledR}$ card which is detailed in Figure (3), this card is formed by 24 samples that include primary colors, compound colors and neutral or gray samples; their values CIE$\mathrm{L} * \mathrm{a} * \mathrm{~b} *$ for D50 and RGB under D65 illuminant are known. To make the analysis, 5 samples were taken into account, which, according to Figure (3) were the holograms of the colors: 13, 14, 15, 16 and 17; it means, the colors: blue, green, red, yellow and magenta.

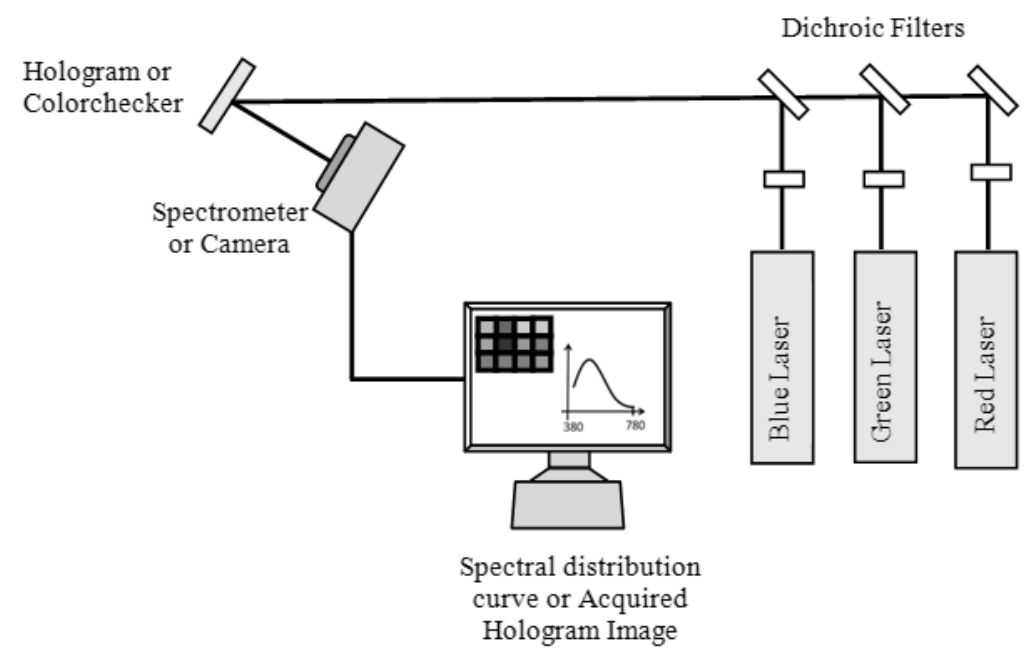

Figure 2. Schematic drawing for measuring color with spectrometer or camera

With the $\mathrm{P}(\lambda)$ reflectance measured, the XYZ and CIE-L*a*b* values of the holograms were calculated through (1) and (2). The configuration for measuring color on the physical card is the same like the used for the hologram, the illumination, the measurement and calculating method are also the same. The color difference was found through (3), where the theoretical CIE-L*a*b* values are the reported by the manufacturer of the card, or, they also could be the CIE-L*a*b* values found for the physical card illuminated with the lasers; in both cases, the experimental values are the found ones for the hologram through the mentioned method.

\subsection{Measurement color arrangement with RGB camera}

This arrangement is very similar as the one of Figure (2), the only difference is the sensor for the measurements, which, in this case is a Thorlabs DCC1645C CMOS RGB Camera. The samples are the same as the used for the spectrometer, it means, the hologram of the Colorchecker card and the physical card under the illumination of three RGB lasers. The images of the holograms were captured for the samples 13 to 17, their RGB values were measured for the linearization and characterization process in Matlab ${ }^{\mathbb{B}}$, then, the CIE-L*a*b* values were calculated from (2). The color difference $\Delta \mathrm{E}$ was calculated in the same way as for the spectrometer. 


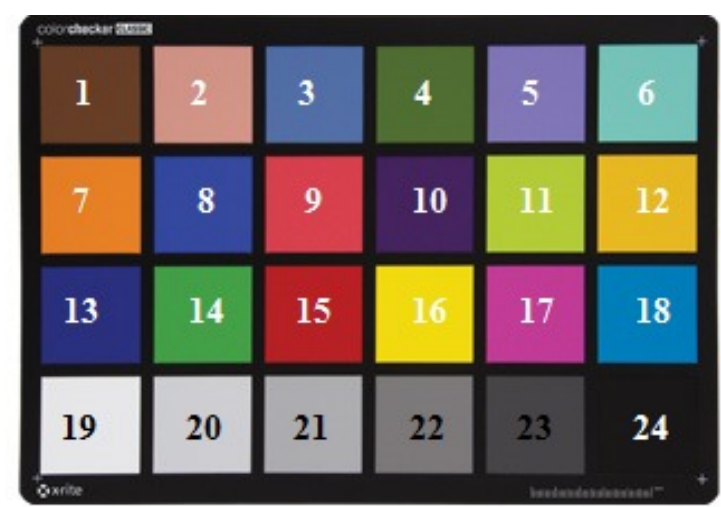

Figure 3. Macbeth Colorchecker $\mathrm{Mini}^{\circledR}$ with detailed numbered samples ${ }^{12}$.

\section{RESULTS}

In the following figure, the images of the physical card and their respective holograms acquired with the CMOS-RGB camera are shown.

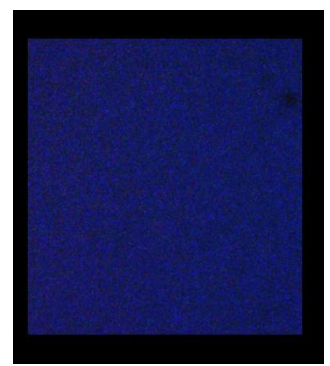

13

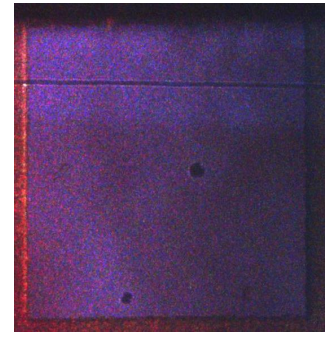

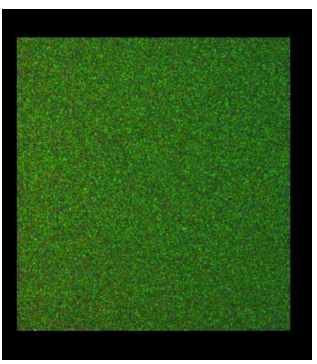

14

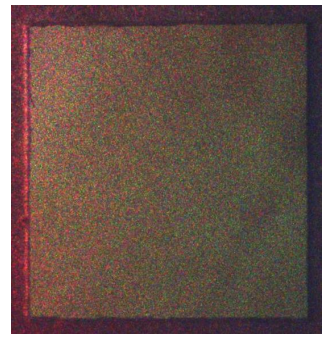

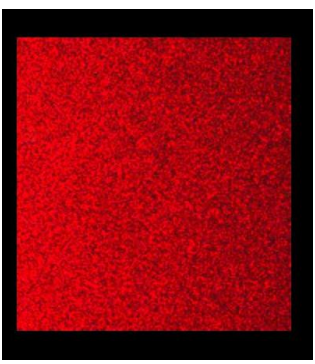

15

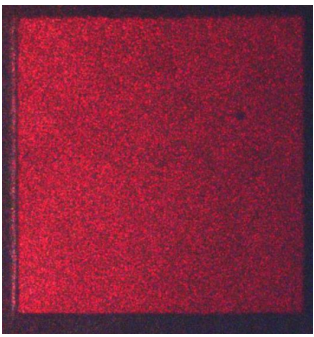

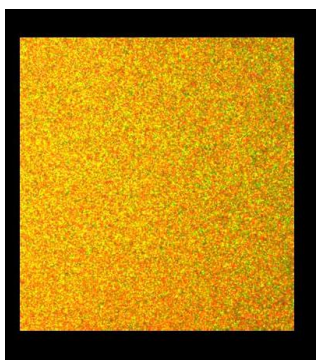

16

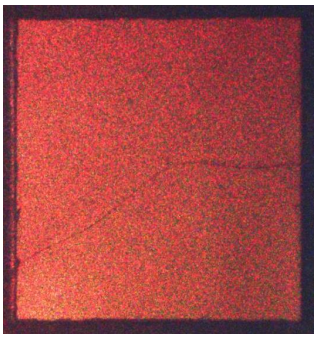

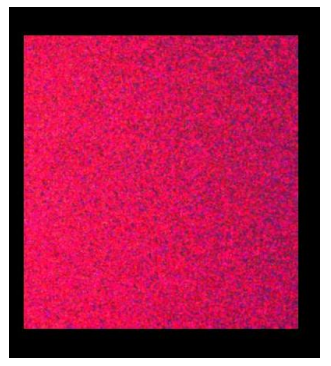

17

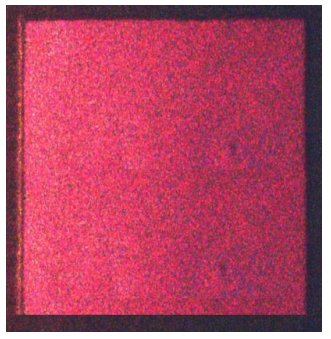

Figure 4. (a) Upper: Acquired images for the physical card with RGB laser illumination. (b) Acquired images for the hologram under the same illumination.

The color differences presented in Table 1 have been obtained between the measurement made on the hologram illuminated with the RGB lasers as experimental values and the reported ones by the card manufacturer as theoretical values illuminated under a D50 illuminant, the measured values for the physical card illuminated with RGB lasers were also used as theoretical values and they are shown in Table 1 too. These measurements are presented for the spectrometer and for the linearized-characterized camera as for the characterized-non linearized camera. It is notice that the highest color differences obtained with the spectrometer correspond to the colors 13 and 17 which are samples blue and magenta; that is because of the low capacity in the hologram to reproduce these colors. 
Table 1. Color differences between the CIE-L*a*b* experimental and theoretical coordinates calculated with spectrometer and camera.

\begin{tabular}{|c|c|c|c|c|c|c|}
\hline \multirow{3}{*}{ Sample } & \multirow{2}{*}{\multicolumn{2}{|c|}{$\Delta \mathrm{E}$ (Spectrometer) }} & \multicolumn{4}{|c|}{$\Delta \mathrm{E}$ (Camera) } \\
\hline & & & \multicolumn{2}{|c|}{ With linearization process } & \multicolumn{2}{|c|}{$\begin{array}{c}\text { Without Linearization } \\
\text { process }\end{array}$} \\
\hline & D50 & RGB Laser & D50 & RGB Laser & D50 & RGB Laser \\
\hline 13 & 106,71 & 78,81 & 47,49 & 23,73 & 40,92 & 16,29 \\
\hline 14 & 48,39 & 24,20 & 44,25 & 26,34 & 40,11 & 18,70 \\
\hline 15 & 45,69 & 57,81 & 19,15 & 5,28 & 29,66 & 11,21 \\
\hline 16 & 20,32 & 13,73 & 73,50 & 64,63 & 61,83 & 52,91 \\
\hline 17 & 74,52 & 59,71 & 44,45 & 28,43 & 42,75 & 31,98 \\
\hline
\end{tabular}

The differences between the chromaticity coordinates of the reproduced object and their theoretical values are represented in a 2D graphic which represents the $\mathrm{a}^{*} \mathrm{~b}^{*}$ plane, these differences are drawn through vectors that correspond to the distance between the theoretical and experimental chromaticity coordinates, these last ones are of the reconstructed object. In Figure (5) these differences are represented for the mentioned samples (13 to 17), the theoretical values are the finish points of the vectors and the experimental values are the starting points, it means, the measured values for the reconstructed hologram. The assigned number to the vectors is the same for each sample; in this figure the vectors for the linearized camera and for the spectrometer are drawn.

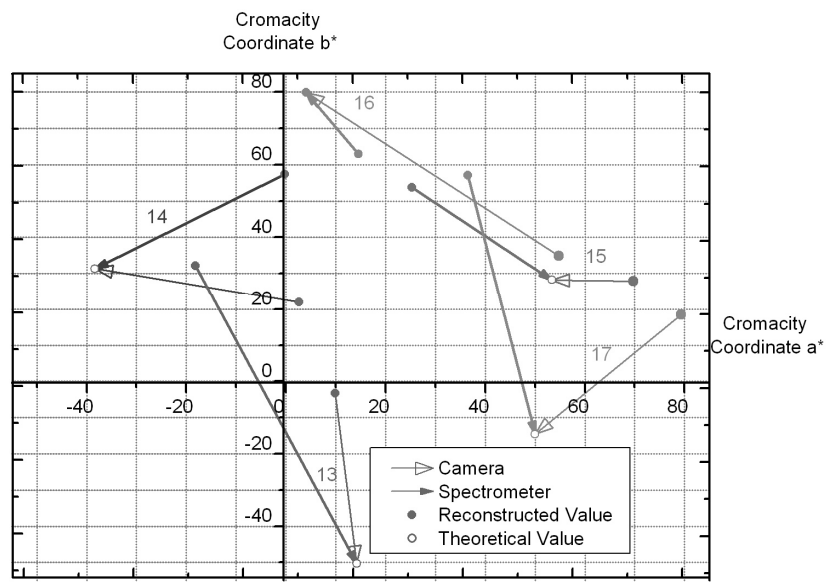

(a)

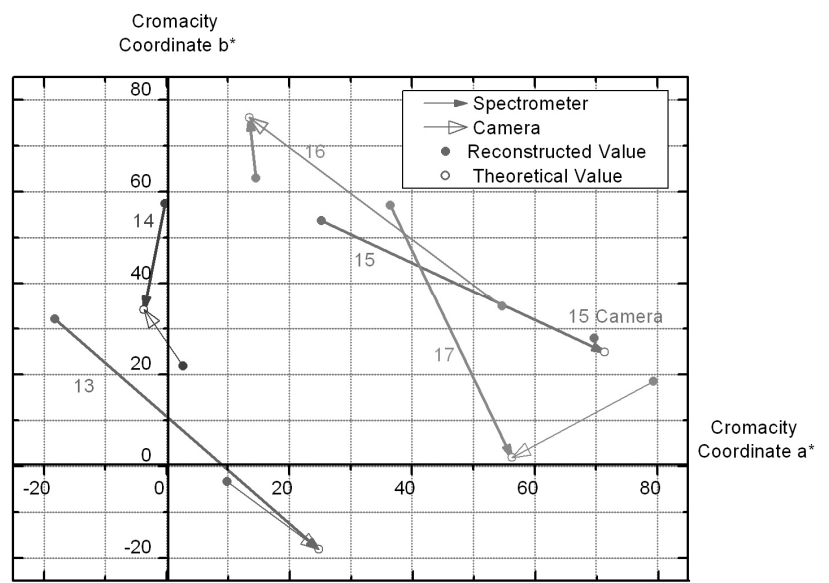

(b)

Figure 5. (a) Comparison between the differences of chromaticity coordinates measured with the spectrometer and with the linearized-characterized camera with the theoretical values reported by the manufacturer card (D50 Illuminant). (b) Differences for the spectrometer and the linearized-characterized camera as experimental values with chromaticity values for the physical card as theoretical coordinates.

According to the last figures, the chromaticity color difference is smaller for the samples when are measured with the camera instead of the spectrometer. According to Table 1, it is observed that for the camera as a measuring method, the non-linearized values have a better behavior than the linearized. Specifically the sample number 15 , which is the red one, presents the smallest difference for both methods: camera and spectrometer; this behavior is expected because of the spectral composition of the reconstructed image, which presents a red coloration in all the samples and it can be seen in Figure (1).

Although the camera seems to be a good method for measuring color in holograms, the parameters of the camera have to be characterized and checked in order to get better measurements and having more concluding reasons to define the best 
method. That is because of the results observed on the graphics of Figure (6), in which the gains of the channels have to be modified because of the low RGB values of the camera for the hologram compared with the RGB values reported for the manufacturer of the card for the same samples under a D65 illuminant.

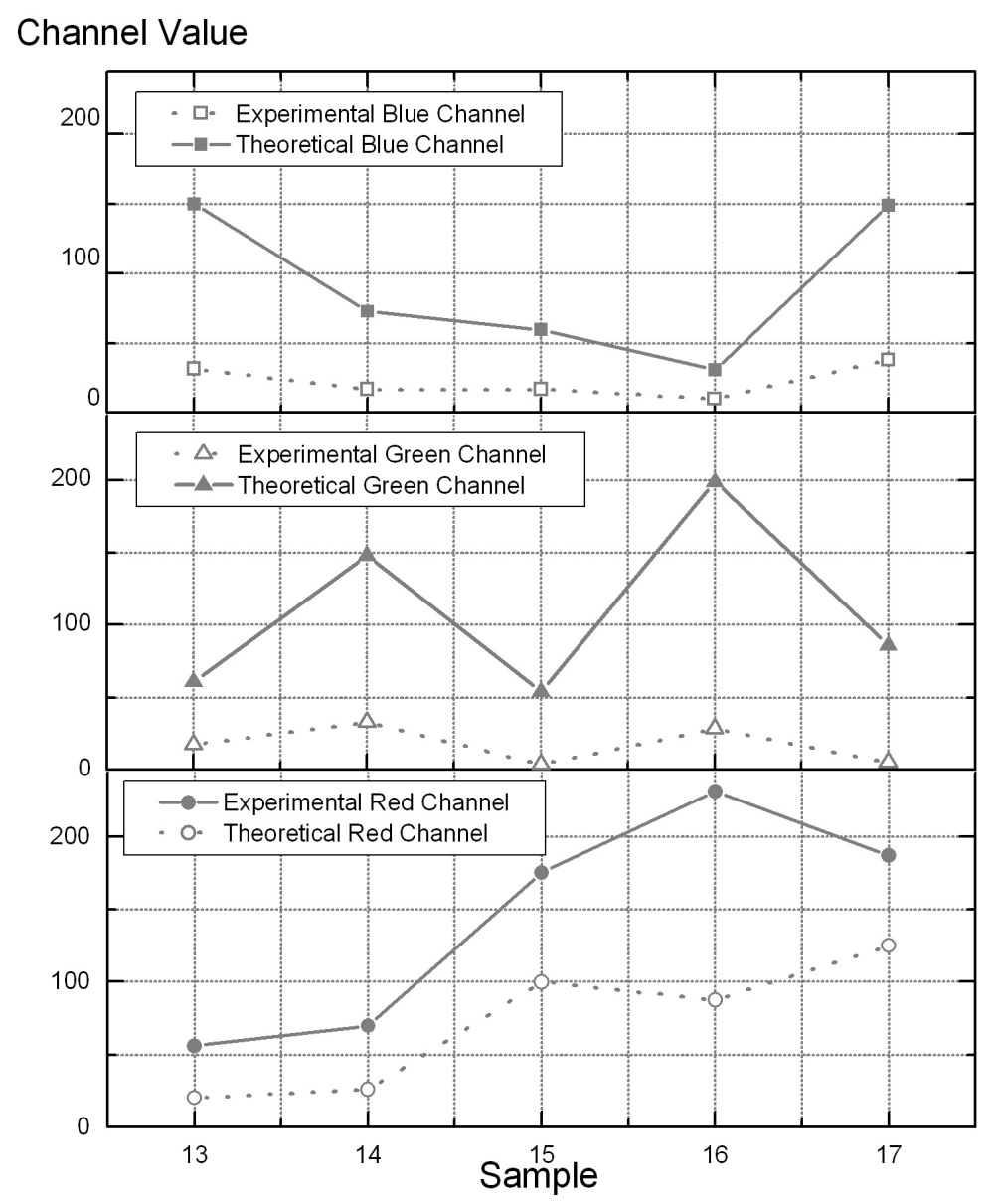

Figure 6. Comparisons between the RGB values of the hologram illuminated with the lasers versus the RGB values of the physical card reported for the manufacturer with a D65 illuminant.

\section{CONCLUSIONS AND FUTURE WORK}

It was found that the method that works the best is the characterized camera, which is because of the color difference calculated for this sensor which is smaller than for the spectrometer.

By making a comparison between the calculated color differences taking as theoretical values the CIE-L*a*b* coordinates reported by the manufacturer of the card with D50 illuminant with the differences calculated taking as theoretical values the card illuminated with RGB lasers, and as experimental values the CIE-L*a*b* coordinates of the reconstructed hologram illuminated with the same lasers, it was found that taking as theoretical values the card illuminated with lasers reported the smaller color differences; that was something to expect because of the selection of the illuminant which is a determinant factor for the color measurement.

The reported color differences with camera and spectrometer depend also on the evaluated sample: the recorded and reconstructed hologram with RGB lasers present a high spectral composition to the red colors, which is why the smallest color differences were found for the 15 sample which is a red color. It is important to notice that the low reproducibility of the color is related to the final characteristics of the hologram, regardless the evaluated measurement methods, because both of them were consistent for the color difference of the same sample. 
By evaluating the obtained results with the camera as a method of color measurement, it was found that the linearized camera presented bigger color differences compared to the non-linearized camera.

In general, for the camera, the experimental RGB values of the hologram presented lower values by comparing them with the RGB values of the same samples reported by the manufacturer of the card; which can be seen in the low intensity of light that comes from the hologram; as a special case, it was found that the green channel $(\mathrm{G})$ is the lowest of the three channels, which affects the measurement in samples who have a high composition to these spectral wavelengths, that was observed in the sample number 16 that corresponds to a yellow color, and presented the highest color difference for the camera as a color measurement method, which means that the green channel gain is too low while the red channel gain is too high.

In order of having a closer comparison to the theoretical values of the card reported by the manufacturer, it is important for future works, the use of a D50 or D65 illuminant in the color measurement process of holograms, which is achieved by obtaining holograms with higher diffraction efficiency. For the measurements with the camera, the transformation matrix has to be calculated under physical samples different to the recorded ones, in order to know how does it affect the CIE-L*a*b* values of the hologram when they are calculated under this kind of matrix.

\section{ACKNOWLEDGMENTS}

These results were obtained in the investigation project that was financially supported by the Investigation Direction of EAFIT University (Colombia). Project code 435-000038.

\section{REFERENCES}

[1] Hubel, P. M. and Solymar, L., "Color reflection holography: theory and experiment," Appl. Opt. 30(29), 4190-4203 (1991).

[2] Yang, F., Murakami, Y. and Yamaguchi, M., "Digital color management in full-color holographic three-dimensional printer," Appl. Opt. 51(19), 4343-4352 (2012).

[3] Noguchi, M., "Color reproduction by multicolor holograms with white-light reconstruction," Appl. Opt. 12(3), 496499 (1973).

[4] Martínez, F. M., Capilla, P. and Pujol, J., [Diseño de un Colorímetro Triestímulo a partir de una cámara CCDRGB], UniversitatPolitècnica de Catalunya, Departamentd'Òptica i Optometria, 137-155 (2002).

[5] Bass, M., Van Stryland, E.W., Williams, D.R. and Wolfe, W.L., [Handbook of Optics: Fundamentals, Techniques and Design], McGraw-Hill, United States, Volume 1, 26.1-26.54 (1995).

[6] Bjelkhagen, H.I., and Mirlis, E., "Color holography to produce highly realistic three-dimensional images," Appl. Opt. 47, A123-A133 (2008).

[7] Peercy, M.S., and Hesselink, L., "Wavelength selection for true-color holography," Appl. Opt. 33(29), 6811-6817 (1994).

[8] Kubota, T.,Takabayashi, E., Kashiwagi, T., Watanabe, M. and Ueda, K., "Color reflection holography using four recording wavelengths," Proc. SPIE 4296, 126-133 (2001).

[9] Westland, S. and Ripamonti, C., [Computational Colour Science using MATLAB], Jhon Wiley \& Sons, Chichester, UK, 127-140 (2004).

[10] Westland, S., Cheung, V. and Thomson, M., "Accurate estimation of the nonlinearity of input/output response for color cameras," Color Research \& Application 29(6), 406-412 (2004).

[11] Velásquez, D., [Estudio Experimental de Medios de Registro para la Obtención de Hologramas a Color], Universidad EAFIT, Medellín, Colombia, 241-248 (2011).

[12] Xritephoto, "Colorchecker Classic,", http://xritephoto.com/ph_product_overview.aspx?id=1192 (28 June 2013). 